\title{
Disentangling homeologous contigs in allo-tetraploid assembly: application to durum wheat
}

\author{
Vincent Ranwez ${ }^{*}$, Yan Holtz², Gautier Sarah², Morgane Ardisson², Sylvain Santoni², Sylvain Glémin³, \\ Muriel Tavaud-Pirra', Jacques David ${ }^{1}$ \\ From Eleventh Annual Research in Computational Molecular Biology (RECOMB) Satellite Workshop on \\ Comparative Genomics \\ Lyon, France. 17-19 October 2013
}

\begin{abstract}
Background: Using Next Generation Sequencing, SNP discovery is relatively easy on diploid species and still hampered in polyploid species by the confusion due to homeology. We develop HomeoSplitter; a fast and effective solution to split original contigs obtained by RNAseq into two homeologous sequences. It uses the differential expression of the two homeologous genes in the RNA. We verify that the new sequences are closer to the diploid progenitors of the allopolyploid species than the original contig. By remapping original reads on these new sequences, we also verify that the number of valuable detected SNPs has significantly increased.

Thirty accessions of the tetraploid durum wheat (Triticum turgidum, A and B genomes) were sequenced in pooled cDNA libraries. Reads were assembled in a de novo durum assembly. Transcriptomes of the diploid species, Aegilops speltoides (close B genome) and Triticum urartu (A genome) were used as reference to benchmark the method.

Results: HomeoSplitter is a fast and effective solution to disentangle homeologous sequences based on a maximum likelihood optimization. On a benchmark set of 2,505 clusters containing homologous sequences of urartu, speltoides and durum, HomeoSplitter was efficient to build sequences closer to the diploid references and increased the number of valuable SNPs from 188 out of 1,360 SNPs detected when mapping the reads on the de novo durum assembly to 762 out of 1,620 SNPs when mapping on HomeoSplitter contigs.
\end{abstract}

Conclusions: The HomeoSplitter program is freely available at http://bioweb.supagro.inra.fr/homeoSplitter/. This work provides a practical solution to the complex problem of disentangling homeologous transcripts in allo-tetraploids, which further allows an improved SNP detection.

\section{Introduction}

Unravelling genome diversity allows addressing basic evolutionary questions as well as giving tools for applied sciences such as plant breeding and livestock management. Single nucleotide polymorphism (SNP) is now a common avenue since Next Generation Sequencing (NGS) technology provides a rapid, cheap and direct access to the genome. In a close future, diversity surveys, high-density mapping, genome wide association studies or genomic selection will be democratized for non model species or orphan crops [1].

\footnotetext{
* Correspondence: Vincent.Ranwez@supagro.inra.fr

'Montpellier SupAgro, UMR AGAP, F-34060 Montpellier, France

Full list of author information is available at the end of the article
}

SNP discovery relies on mapping re-sequencing data of a diversity panel on a reference sequence. As complete genome sequences will still require a lot of efforts and international consortia, e.g., the International Wheat Genome Sequencing Consortium (IWGSC, http://www. wheatgenome.org), sequencing a reduced but repeatable portion of the genome appears as a provisory amenable approach. Reduction of genome complexity can be achieved via sequencing cDNA obtained in standardised conditions (RNAseq) [2,3]. Difficulties for the use of RNAseq for SNP discovery come from alternative splicing, differential expression between genes leading to poor coverage of lowly expressed genes, weak evolutionary signal for detecting paralogous genes and transcription errors 
[4]. Nevertheless, this approach has though proven its efficiency to genotype few individuals on several thousands of genes for non model organisms [5] and to produce SNP data base in many species $[2,3]$.

Plants in contrast to animals are often of recent polyploid origin [6,7]. In allopolyploids, two or more subgenomes are present and SNP discovery and genotyping using cDNA is complicated by the parallel expression of homeologous copies of the same genes. Observed sequence variation may be due to divergence between homeologous copies or to intra-genome (homologous) allelic polymorphism. Reads of cDNA homeologous copies are thus frequently assembled in the same reference gene (either from de novo reference assembly or on the whole genome sequence) and genotypes commonly show excess of spurious heterozygous sites [3]. Genomic sequences are submitted to the same confusion effect [8]. The SNPs with excess of heterozygosity are usually simply discarded [8], lowering down the yield in workable SNPs. This specific situation of allopolyploidy is superimposed to other sources of errors also existing in diploid species such as undetected paralogy or copy number variation [1]. Developing a good method for tackling this issue is crucial for important allo-polyploid crops (potato, durum and bread wheat, cotton, canola, tobacco and peanuts [1]).

Computational methods for coping with paralogy induced by allopolyploidy are dealing first with assembly stringency. If the divergence between the two subgenomes is tight, a lot of confusion will still be present in assembly. Increasing stringency also leads to a reduced length of de novo assembled contigs and to the possible, undesirable, separation of allelic variants. The use of the diploid progenitors may be of great help for the identification of sub-genomes specificity [9]. When this resource is not available (when at least one of them is not available or identified) or when the diploid assembly does not cover the whole transcriptome of the allo-polyploid target species, there is no real satisfying method. Identifying homeologous de novo contigs and splitting them into two new sequences could help resolving the issue of heterozygous excess due to homeologous confusion. Remapping reads on these two new sequences may provide a more clearcut discrimination of valuable homologous SNPs.

\section{State of the art}

The problem of reconstructing the two homeologous copies merged in a single contig can be seen as a variant of the phasing problem that aims at reconstructing the different haplotypes merged in a single contig. This latter problem has been extensively studied since the seminal work of Clark in 1990 [10]. Existing algorithmic solutions can be grouped in two main categories: i) the genotyping based approaches that infer haplotypes based on the genotyping of several accessions and ii) the cooccurring based approaches that infer haplotypes based on nucleotide co-occurrence on the same sequenced fragment. Those two strategies will be briefly presented here with respect to their potential use to separate homeologous copies (see [11] for a review about their relative efficiencies).

Genotyping based approaches mostly rely on population genetics theory to identify a set of haplotypes in a parsimonious [12-14]; maximum likelihood [15] or bayesian framework [16,17]. Such approaches are hardly adaptable to disentangle homeologous copies since in this latter case i) the mix of homeologous copies will bias the genotyping inference on which they rely ii) their underlying model assume coalescences of haplotypes within a single locus whereas homeologous copies result from a single duplication event. For instance, in the simplest Haplotype reconstruction described by Clark in 1990 [10]; the algorithm starts building reliable haplotypes by identifying individuals with no (or only few) heterozygous sites whereas it is precisely the reverse situation (heterozygous excess) that suggests homeology.

Phasing method based on nucleotide co-occurrence in the same reads (or ESTs) mostly ignore the underlying biological model and rely on combinatorial and graph theory to tackle the problem [18-20]. The fact that two nucleotides appear on the same reads is a strong indication that they belong to a same haplotype. This indication can however be erroneous on low coverage regions due to sequencing errors. Moreover, the fact that reads come from the same accession is not taken into account, so that even if an accession is homozygous CC and TT at two distant sites, phasing is ignored unless some reads overlap the two sites for this accession. In addition, SNP density must be sufficiently high to ensure that many reads contain two or more SNPs all along the contig [21]. Such methods are thus adapted for high coverage sequencing with long reads and/or high SNP density [11]. For all those reasons, they are often used as post-processing of genotyping basedphasing as for instance in Haplotype Improver [18]. A recent work uses a different approach that simultaneously assembles reads and predicts haplotypes using colored De Bruijn graphs [22], a dedicated variant of the De Bruijn graphs [23]. The associated CORTEX software [24] uses individual information to predict haplotypes. Disentangling homeologous copies can be seen as a quite similar problem except that, in allo-polyploid species, no easy solution exists to allocate a distinct colour to reads coming from the two homeologous copies with the exception of chromosome sorting [25].

In this paper, we propose a solution, dedicated to allotetraploid species, that uses the nucleotide counts observed at each site, which is much more informative than using just accession genotypes but much more lightweight than 
using full read information. This allows us to design a fast dedicated solution that identifies contigs for which a heterozygous excess may sign the assembly of two homeologous/paralogous copies. On these contigs, we propose a likelihood model-based method to rebuild mixed contigs in two new sequences based on their differential expression, a largely documented phenomenon on homeologous copies [26-28]. We test the new sequences for their distances to the diploid progenitors of the allo-tetraploid species and verify their efficiency to map properly reads and to provide a significant increased amount of new valuable SNPs compared to de novo mapping method.

The method is implemented in the HomeoSplitter software, tested and evaluated on durum wheat (Triticum turgidum L.), an allo-tetraploid inbreeding species and is validated on the reference transcriptomes of its two diploid progenitors.

\section{Material}

\section{Durum wheat genomes}

Allo-tetraploid wheats (T. turgidum L.) originate from the spontaneous hybridization of two ancestral diploid species ( $2 \mathrm{n}=4 \mathrm{X}=28$, A and B genomes). The current descent of its A progenitor has been identified as the diploid T. urartu [29] which genome is still lowly differentiated from the A genome of durum. Aegilops speltoides is the most closely related extant species to the $B$ genome of tetraploid wheat [30], the real ancestor of the $B$ genome being either extinct or not yet discovered. The divergence of the Triticum/ Aegilops alliance is approximately dated between 2.5 and 4.5 million years ago [31]. The origin of $A B$ tetraploid wheat was reported to have originated ca. 0.36 million years ago [32].

\section{Accessions, cDNA extraction and preparation}

The diploid reference transcriptomes were assembled from the cDNA libraries obtained on a Triticum urartu accession ( $d v 1792$, kindly provided by Pr J. Dvorak, UC Davis) and an Ae. speltoides var. speltoides accession from Turkey (USDA PI 542268, http://www.ars-grin. gov/npgs/). These two lines will be further called urartu and speltoides accessions.

The 30 durum accessions analysed were issued from single seed descents of plants sampled in a base broadening population of durum wheat (see additional file 1). The averaged observed heterozygosity of the 30 descents was 0.022 on 30 microsatellite loci. Taking allelic frequencies into account, the fixation index, Fis [33] was estimated at 0.95 on the whole sample (data not shown). These 30 accessions will be called durum wheat accessions.

For each accession, we obtained sequence data by RNAseq procedure, mainly consisting in mRNA extraction and purification, libraries construction, mixing, and sequencing using the Illumina mRNA-Seq, paired-end indexed protocol on a HiSeq2000 sequencer. The protocol is detailed in additional file 2.

\section{Reads assembly and mapping}

The whole pipeline described in this section is schematically summarized in Figure 1 and detailed in the additional file 3. Urartu and speltoides accessions were assembled in respectively urartu and speltoides contigs, and durum accessions were assembled in de novo contigs. Durum reads were thus mapped on three different references: 1) on urartu and speltoides de novo contigs giving "diploids SNPs", 2) on durum de novo contigs, giving "de novo SNPs", 3) on the newly recomposed contigs ("HomeoSplitter contigs") using HomeoSplitter (see below) giving "HomeoSplitter SNPs".

\section{Benchmark constitution}

To evaluate and validate our HomeoSplitter approach, we clustered de novo and diploid contigs using CAP3 [34]. Though CAP3 is not initially designed for sequence clustering it works particularly well in our case since sequences to be clustered are highly similar, it also has the advantage of directly providing us with an alignment of those sequences. We then kept only clusters containing simultaneously one contigs of urartu, one of speltoides and one or two of durum having at least 100 nucleotides of overlap with the diploid contigs.

\section{Methods}

HomeoSplitter aims at identifying contigs that result from a mixing of reads coming from homeologs and replacing each of those contigs by two new contigs (one per homeolog). First, HomeoSplitter identifies problematic sites and contigs based on their excess of observed heterozygosity. Indeed, at sites where the apparent polymorphism is actually due to divergence between the subgenomes, an excess of heterozygosity is expected. Then, HomeoSplitter disentangles the two homeologous contigs based on the potential differential expression between the two homeologous copies of a given gene in the same accession. As the read counts may differ between homeologs according to their expression, the basic idea is that the most numerous nucleotides at all heterozygous sites of a given contig is likely to come from the most expressed homeolog. Taking into account the fact that the differential expression can vary among accessions is somehow more complex. HomeoSplitter tackles this problem by searching for the two contigs, obtained from the original one by modifying its problematic sites, which maximise the likelihood of the observed nucleotide frequencies under the assumption of differential expression between homeologs. 


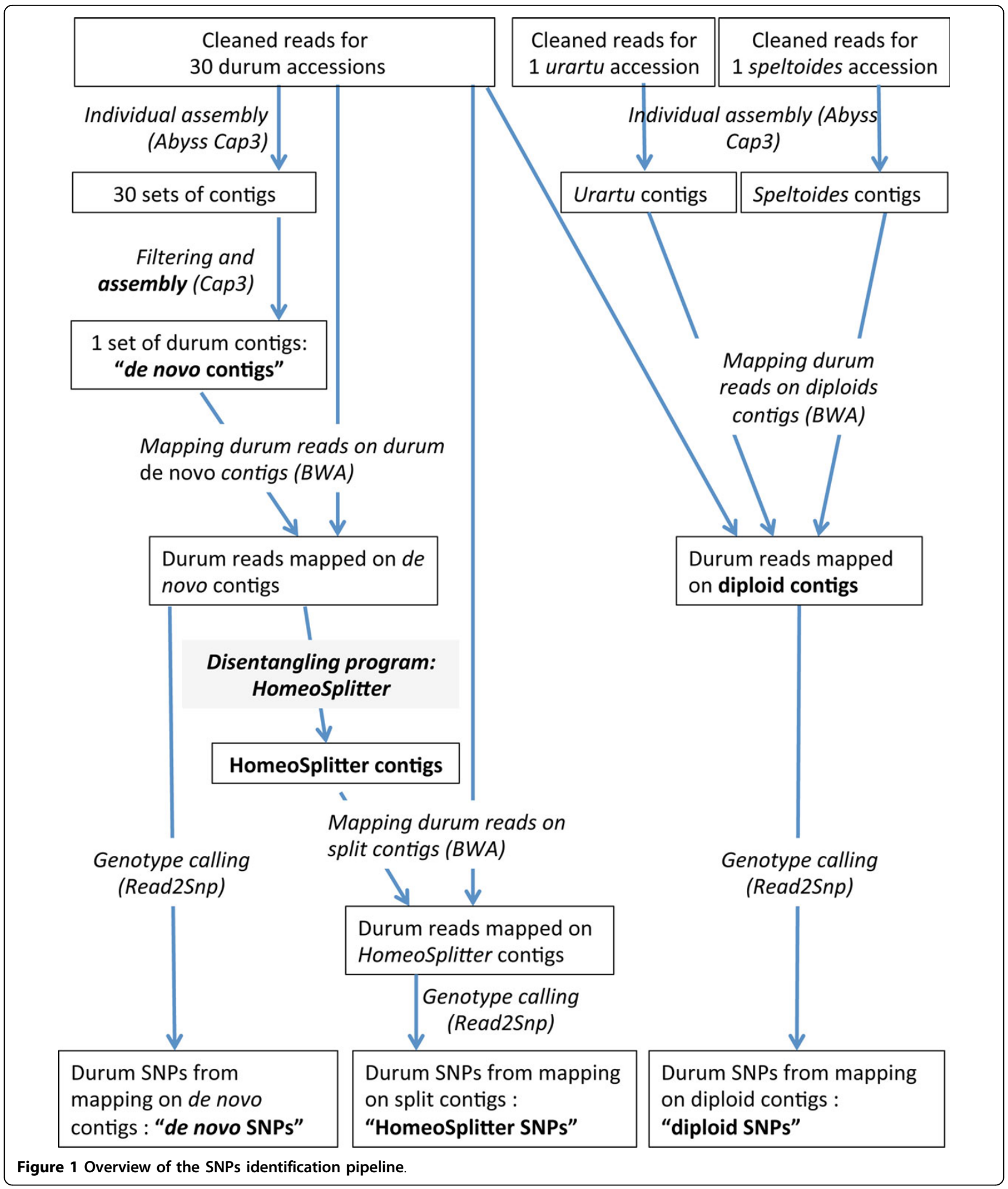

\section{Notations}

Let $N b$ be the array of $M x N x 4$ cells containing nucleotide counts observed at $N$ sites for $M$ distinct accessions, such as $N b[a][s][1]$ (resp. $N b[a][s][2], N b[a][s][3]$ and $N b[a][s][4]$ ) gives the observed number of nucleotide A (resp. C, G and $\mathrm{T}$ ) of the $s^{\text {th }}$ considered sites of the $a^{\text {th }}$ accession (with $1 \leq s \leq N$ and $1 \leq a \leq M$ ). Figure 2 provides example of such nucleotide counts. 


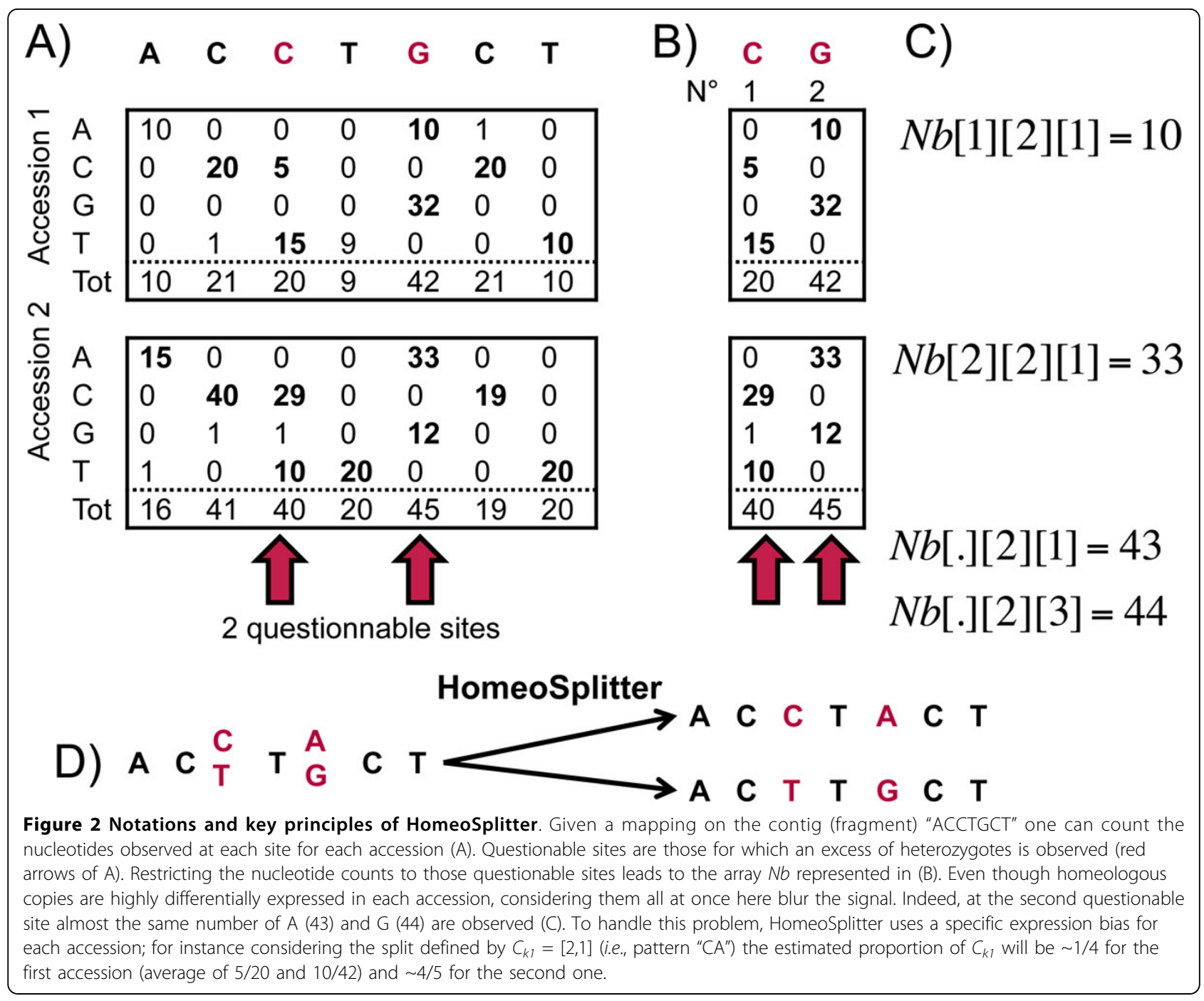

Let $M f 1 \_N b$ (resp. $M f 2 \_N b$ ) be the $M x N$ array of the indices of the most frequent (resp. second most) nucleotides observed in $\mathrm{Nb}$ for each accession. For instance, if $N b[2][1]=[0,29,1,10]$ as in Figure 2B); then $M f 1 \_N b[2]$ [1] $=2(\mathrm{C}$ is the most frequent nucleotide at the first site in the second accession) and $M f 2 \_N b[2][1]=4$ (i.e., T).

Note that counts from $\mathrm{Nb}$ can be considered globally (considering all accessions simultaneously) or locally for a given accession. To handle those different cases we will use the "." symbol to denote the fact that we sum over all possible value for this unspecified parameter (marginal sums). Using this convention then $\mathrm{Nb}[].[\mathrm{s}][$. denotes the total number of nucleotides observed at site $\mathrm{s}$ for all accessions; $\mathrm{Nb}[].[\mathrm{s}][1]$ denotes the total number of nucleotide A observed at site $\mathrm{s}$ for all accessions and so on (see Figure 2) C). Using analogous convention we denote by $M f 1 \_N b[].[s]$ (resp. $M f 2 \_N b[].[s]$ ) the index of the most frequent nucleotide at site $s$ over all accessions.

\section{Detecting questionable sites and contigs}

We questioned the polymorphic sites obtained from the mapping of reads on the de novo contigs (see Figure 2). First, a site $s$ is here considered to be heterozygous for one accession $a$ if at least two different nucleotides $n_{1}$ and $n_{2}$ are each observed at least 5 times (e.g., sites pointed by red arrows in Figure 2A). More formally, those sites are those such as:

$$
\exists n_{1,}, n_{2} \mid 1 \leq n_{1}<n_{2} \leq 4 ; N b[a][s]\left[n_{1}\right] \geq 5 ; N b[a][s]\left[n_{2}\right] \geq 5
$$

We then define a site as questionable if it is found heterozygous in at least one eighth of the genotyped individuals out of the 30 possible durum accessions. These two parameters can be tuned by users according to their biological datasets. The $5 \mathrm{X}$ coverage threshold limit the impact of sequencing errors and the $1 / 8$ threshold is adapted to our almost fixed accessions. We consider the presence of questionable sites as an indication that this contig is indeed a mix of homeologous genes. 
A contig is hence considered to be questionable if it contains at least one questionable site and has an average coverage of at least $5 \mathrm{X}(\mathrm{Nb}$ [.] [.] [.] $/ \mathrm{N} \geq 5)$. Our approach assumes that each questionable contig mix reads from the two genomes of the studied tetraploid and could be hence split in two new contigs, identical for all sites but the questionable ones. Defining the split into two new contigs is thus equivalent to defining the two series of nucleotides observed at the questionable sites.

\section{Splitting questionable contigs}

Having computed $N b, M f 1 \_N b$ and $M f 2 \_N b$ arrays for the $N_{q}$ questionable sites of a contig $C_{k}$, we can now try to disentangle problematic contigs by splitting them. A variant $C_{k 1}$ of $C_{k}$ is defined through an array of nucleotide of size $N_{q}$, that defines the pattern used to replace nucleotide of $C_{k}$ at questionable sites (e.g., $C_{k 1}[2]=1$ indicates that the second questionable site of $C_{k}$ will be replaced by an $\mathrm{A}$ in the new contig $C_{k 1}$ ). As we assume that questionable contigs are a mixture of two homeologous contigs, the complementary contig of $C_{k 1}$, denoted as $C_{k 2}$ is obtained by replacing the questionable nucleotides of $C_{k}$ by the most frequent nucleotides at this position once the nucleotide used in $C_{k 1}$ has been excluded. More formally,

$$
C_{k 2}[s]=\left\{\begin{array}{c}
M f 1_{-} N b[.][s] ; \text { if } M f 1 \_N b[.][s] \neq C_{k 1}[s] \\
M f 2 \_N b[.][s] ; \text { otherwise }
\end{array}\right.
$$

A simple solution to split questionable contigs is to use the most frequent nucleotides observed at all questionable sites to build $C_{k 1}$ (i.e., $C_{k 1}[s]=\mathrm{M} f 1_{-} N b[].[s]$ ) and, hence, to use the second most frequent nucleotides to build $C_{k 2}$ (note that by construction, this majority sequence corresponds to the de novo contig $C_{k}$ on which reads have been mapped). We call this first method the MajoritySplitter, this method assumes that the two homeologous genes are differentially expressed so that the most frequent nucleotides are likely to belong to the same homeologous sequence.

When considering several accessions, if the expression bias between the two copies is similar in all accessions, the signal is reinforced. On the other hand, if this bias varies from one accession to another, the final signal can be blurred when all individual data are merged altogether. For instance, if accession 1 has $1 / 3$ of its reads from the genome $\mathrm{A}$ and $2 / 3$ from the genome $\mathrm{B}$ when accession 2 has $2 / 3$ of reads from genome $A$ and $1 / 3$ from $B$ then on average, each genome provides $50 \%$ of the reads and there is no average differential expression. In such cases the majority sequences (and de novo assembled contig) can be chimeric contig mixing genome A and B (see Figure 2 for such an example). To ensure that individual signals add synergistically and not antagonistically, we computed the likelihood of the possible splits by explicitly using expression bias for every accession in each contig.

\section{Computing the likelihood of a split}

The likelihood of the split defined by a pattern $C_{k 1}$ combines the likelihood of the pattern $C_{k 1}$ and the likelihood of its complement pattern $C_{k 2}$. The idea is that together they should provide a likely explanation of the nucleotide distribution observed at each questionable site. The ratio of the two homeologous gene copies in the sequenced cDNA is assumed to have influenced the ratio of $C_{k 1}, C_{k 2}$ reads that have been sequenced. The absolute number of reads sequenced at each questionable site may vary among sites but the ratio between those of $C_{k 1}, C_{k 2}$ is assumed to be roughly constant for a given accession all along the contig. We estimate the proportion of $C_{k 1}$ specific to an accession $a$, denoted as $p_{1}[a]$, as the average of this proportion along all well covered sites:

$$
p_{1}[a]=\underset{\substack{\text { average } \\ 1 \leq n b \text { QuestionableSites }}}{N b[a][s][.] \geq 10}\left(N b[a][s]\left[C_{k 1}[s]\right] / N b[a][s][.]\right)
$$

The expected proportion of $C_{k 2}$, denoted as $p 2[a]$, is then set as $p 2[a]=1-p 1[a]$.

Given $p 1[a]$ and $p 2[a]$ the chance of having the observed nucleotide frequencies follows a binomial distribution so that the probability of site $s$ of accession $a$ given $C_{k 1}$ and $C_{k 2}$ under this model is:

$$
\begin{aligned}
& p\left(N b[a][s] \mid C_{k 1}[s], C_{k 2}[s]\right)=\left(\begin{array}{c}
N b[a][s][\cdot] \\
N b_{k 1 a s}
\end{array}\right) p_{1}[a]^{N b_{k 1 a s}}\left(1-p_{1}[a]\right)^{\left(N b[a]\left[s[[]]-N b_{k l a s}\right)\right.} \\
& \times\left(\begin{array}{c}
N b[a][s][.] \\
N b_{k 2 a s}
\end{array}\right) p_{2}[a]^{N b_{k 2 a s}}\left(1-p_{2}[a]\right)^{(N b b a]\left[s[1] \cdot-N b_{k 2 a s}\right)}
\end{aligned}
$$

with $N b_{k 1 a s}\left(=N b[a][s]\left[C_{k 1}[s]\right]\right)$ the number of occurrences at site $s$ in accession $a$ of the nucleotide proposed in the pattern $C_{k 1}$ at this site; $N b_{k 2 a s}$ the equivalent for $C_{k 2}$, and $N b[a][s][$.$] the total number of nucleotides observed$ at this site for this accession. Note that if $C_{k 1}[s]$ is a nucleotide rarely observed at site $s$, corresponding to a sequencing error, the first term of the product may be quite high, but it will be counterbalanced by the second product term that will be quite low. Hence the necessity to consider both term simultaneously. For instance if there is a single accession and a single questionable site where $49 \mathrm{~A}, 50 \mathrm{C}$ and $1 \mathrm{~T}$ are observed, the first term will be maximal when considering that $C_{k 1}[1]$ is a $T$, since with $p_{1}=1 / 100$ it is highly probable to observe $1 \mathrm{~T}$ among 100 draws, but since it is also highly unlikely to observe only $50 \mathrm{C}$ among 100 draws with $p_{2}=0.99$ this split will be discarded in favour to the much more likely explanation that $C_{k 1}$ [1] is an $A$ and $C_{k 2}$ [1] is a $C$, or vice versa. Note also that these two equivalent solutions $\mathrm{A} / \mathrm{C}$ or $\mathrm{C} / \mathrm{A}$ do not have exactly the same probability under our model, and this will always be the case as soon as there are more than two nucleotides observed at the considered site. 
Finally, we assume independence between sites so that the likelihood of $C_{k 1}, C_{k 2}$ is just the product of site probabilities along sites and accessions:

$$
\operatorname{Lk}\left(C_{k 1}, C_{k 2}\right)=P\left(N b \mid C_{k 1}, C_{k 2}\right)=\prod_{s, a} p\left(N b[a][s] \mid C_{k 1}[s], C_{k 2}[s]\right)
$$

Heuristic used to search for the maximum likelihood split Our HomeoSplitter software offers two different strategies to determine the preferred split. The first one is an (almost) exhaustive search of the most likely split. For each questionable site we considered nucleotides present at least 5 times in at least one accession and test all combinations of those nucleotides. For each site the number of possibilities is thus greater or equal to 2 (otherwise the site will not be questionable) the total number of tested combinations is thus greater than $2^{N_{q}}$ for $N_{q}$ questionable sites. This solution is not tractable for large value of $N_{q}$. Since the model assumes that the two homeologous copies are differentially expressed, it is reasonable to test the splits corresponding to the majority sequence of each accession (i.e., $C_{k 1}[i]=M f 1 \_N b[a][s]$ ). We also consider the split build from the most frequently observed nucleotides at all questionable sites (i.e., the split returned by MajoritySplitter). As several accessions may have identical majority sequences, this leads us to design a heuristic search that tests at most $(M+1)$ splits. As for $N_{q} \leq 10$ the full search was tractable, we compare both solutions on this subset of our benchmark. Since the same split is proposed by both methods in $99.6 \%$ we use the above described heuristic as the default strategy in HomeoSplitter without further attempt to better optimize our search strategy using a more complex strategy.

\section{Split similarity with the $2 \mathrm{X}$ reference sequences}

To assess the performances of our HomeoSplitter method we compare the initial contig with the two resulting contigs supposed to represent the disentangled homeologous copies. This was done using the previously described benchmark, which focuses on durum contigs $C_{k}$ for which similar sequences have been found in each of the two diploid relatives, urartu and speltoides. The consensus sequence of the durum reads mapped on the diploid contigs (Figure 1) provides us with a "golden standard" of an ideal homeologous assembly hence, good splits are expected to have a good similarity with these two reference consensus. For the contig $C_{k}$ those two consensus sequences are respectively denoted as $S_{k}$ and $U_{k}$. To assess whether splits obtained via HomeoSplitter get closer or not to this golden standard, we use the following criteria: with $\operatorname{Sim}$ the similarity between two contigs defined as the percentage of identical nucleotides along their overlap. Note that the length of the considered overlapping stretches may be different with urartu and speltoides but they always spread over at least 100 bp by construction of our benchmark.

As the max included in this formula may bias this measure toward positive values we also compared the performance of HomeoSplitter and MajoritySplitter to a random split of the contig (randomly changing the same number of sites, or randomly changing each questionable site with one of the observed nucleotides at this site).

\section{Genotyping, SNP detection and the Fis fixation index}

The genotyping of each accession was done using reads2snp [35], which estimates the most likely genotype and its associated probability given a prior Fis value of 0.85 . Under complete selfing, Fis should be set to 1 while under panmixia Fis should be set to 0 . In our case, as durum lines were extracted from a mixed mating durum population, Fis was empirically set to 0.85 , based on previous empirical estimates. We then conserve as reliable genotypes only those having, according to read2SNP, a probability greater than 0.99 and called on sites covered by more than ten reads.

At each polymorphic site (SNP), allele frequencies were computed on the set of properly genotyped individuals. From these values, Fis value was computed at each site. If $H_{o b s}$ is the ratio of observed heterozygous individuals among the properly genotyped accessions for this site, then Fis $=1$ - Hobs $/(2 p(1-p))$ where $p$ is one of the allelic frequencies (we kept only the bi-allelic SNPs here). Fis will be used as follows to validate SNPs and determine the efficiency of HomeoSplitter. In case of complete divergence between the two homeologs mixed in a single contig, all the genotypes will be heterozygous and Fis value will be negative (Fis $=-1$ since Hobs $=1$ and $p=$ $1 / 2$ ). A good SNP in an inbred population such as durum wheat will have a Fis value close to 1 (Fis $=1$ when no heterozygous genotype is observed). Intermediate situation may appear when one homeologous copy is monomorphic at one site (e.g., AA) when a derived allele is polymorphic on the other copy (e.g., AA or TT for inbred lines). In a mix contig, if $p$ is the frequency of the $T$ allele, $\sim p M$ accessions will appear as heterozygote AT (actually AATT) and (1-p) as homozygous AA (actually AAAA). In this homeologous/homologous polymorphic situation, Fis $=0$ for $p=1 / 2$. We thus expect Fis values to be distributed around three modes: close to 1 for valuable polymorphic homologous SNPs, close to 0 for a homeologous mix with a homologous polymorphism in one of the copies, close to -1 for SNPs revealing fixed divergence between copies. 
A way to measure the efficiency of HomeoSplitter is thus to compare the distribution of the Fis of the de novo SNPs, HomeoSplitter SNPs and diploid SNPs.

\section{Results and discussion \\ Software availability}

HomeoSplitter is freely available at http://bioweb.supagro.inra.fr/homeoSplitter/ under the French CeCILL licence, which is compatible with the GNU GPL one. The software has been developed in Java and can thus be run on Windows, Linux and Mac Operating Systems. It takes as input an ALR file representing the frequency of each nucleotide in the initial mapping [35] and provides the new set of split contigs in fasta format as output. The SAM format is the de facto standard to represent mapping [36], the software SAM2ALR [35] allows to transform SAM formatted files into the ALR file that HomeoSplitter takes as input. All threshold values used to detect questionable sites and contigs are parameterized and HomeoSplitter also accepts a set of questionable sites as input. Starting from an ALR file containing reads counts for $\mathrm{M}$ accessions over a set of contigs, HomeoSplitter has a time complexity of $O(N M)$ and a space complexity of $O\left(N_{l} M\right)$; with $N_{l}$ the length of the longest contigs and $N$ the sum of contig lengths. These low complexities allow to run HomeoSplitter on standard desktop computer; for instance our dataset of 3,709 contigs was processed in less than four minutes on a MacBook Pro laptop (OS X.6, 4 Go RAM, 2.3 GHz Intel Core i7 CPU).

\section{Considered dataset and benchmark assembly}

The number of cleaned reads varies from 1.3 to 10.8 millions among the 30 durum accessions, with an average of 4.01 millions. Speltoides assembly was built with 20.25 millions cleaned reads and that of urartu with 26.7 millions. Speltoides assembly counts 16,891 contigs (sized by length $>500 \mathrm{bp}$ ) with an average size of 1,193 bp when urartu assembly has 21,907 sized contigs with an average size of $1,351 \mathrm{bp}$. The assembly of the durum reads coming from the 30 accessions yielded 27,820 de novo sized contigs of $1,055 \mathrm{bp}$ on average.

After clustering (CAP3 $90 \%$ of similarity), a total of 40,895 contigs (urartu + speltoides + durum) are present in 13,166 clusters. Durum contigs are present in 11,630 clusters (88\%). The different clustering configurations are as follows: (durum + speltoides + urartu): 6,057 clusters; (durum + speltoides without urartu): 1,899 clusters; (durum + urartu without speltoides): 2,667 and durum alone: 1,007 clusters. Out of the 6,057 (durum+speltoides+urartu) clusters, only a subset of 2,505 clusters validated the conditions required for the benchmark of HomeoSplitter. These 2,505 clusters contain 3,709 durum wheat contigs, among which we kept only the
2,816 having an overlapping of at least $100 \mathrm{bp}$ with simultaneously urartu and speltoides contigs.

\section{Improvement when disentangling contigs using HomeoSplitter}

New contigs proposed by HomeoSplitter are closer to the diploid contigs than was the de novo contig in 2,506 out of 2,816 cases $\left(89 \%\right.$ of $\operatorname{diffSym}\left(C_{k}\right)>0$ ). In $6.2 \%$ they were more distant $\left(\operatorname{diffSym}\left(C_{k}\right)<0\right)$ and in $4.7 \%$ equally close $\left(\operatorname{diffSym}\left(C_{k}\right)=0\right)$. A paired t-test confirms that this difference is evidently highly significant ( $\mathrm{p}$-value $<2 \cdot 10^{-16}$ ). To control for the bias introduced by the max operator in the diffSym measures, we also consider splits obtained by choosing randomly at each questionable site one of the observed nucleotides. Both HomeoSplitter and MajoritySplitter produce significantly better values than this pseudo random splitting strategy ( $\mathrm{p}$-value of a paired t-test $\left.<2.10^{-16}\right)$. Moreover, diffSym values obtained with HomeoSplitter are also significantly better than those obtained by the MajoritySplitter approach (454 cases better, 140 cases worse, $p$-value $<210^{-16}$ ). This result may be explained if the most expressed homeologous copy of one gene is not always the same among durum accessions, either because of genetic difference in gene regulation among accessions or from environmental differences during the growth and the sampling of seedlings.

The distributions of Fis obtained on the three mappings are plotted in Figure 3. Fis varies between -1 and 1 and the three expected modes are effectively observed. Mapping on diploid contigs and HomeoSplitter contigs produce very close results. Compared to the de novo case, using HomeoSplitter significantly reduces the proportion of negative Fis (Fis=-1 mode). This clearly indicates that HomeoSplitter separated correctly completely diverged sites between the two homeologous copies mixed in the original de novo durum contigs. The original contig is often highly similar to one diploid (the most expressed one) but reads from both copies are mapped on it. These cases are revealed by applying HomeoSplitter which splits it in two distinct contigs one being similar to urartu and the other to speltoides. This is illustrated in Figure 4 through the maximum likelihood phylogenetic tree of the different contigs present in the cluster number 6960 . Conversely mapping on HomeoSplitter contigs greatly enhances the number of SNPs with high Fis values, particularly in the class above 0.9. The expected value of Fis of true homologous SNPs in our accessions is expected to be located around ca. 0.95 . This proves that the excess of heterozygosity has been greatly decreased in this mapping compared to the mapping on the de novo durum contigs and that more "homologous" SNPs are now correctly detected. As Fis may vary, we empirically put a threshold value at Fis $=0.6$ to accept a SNP as "homologous". We found 


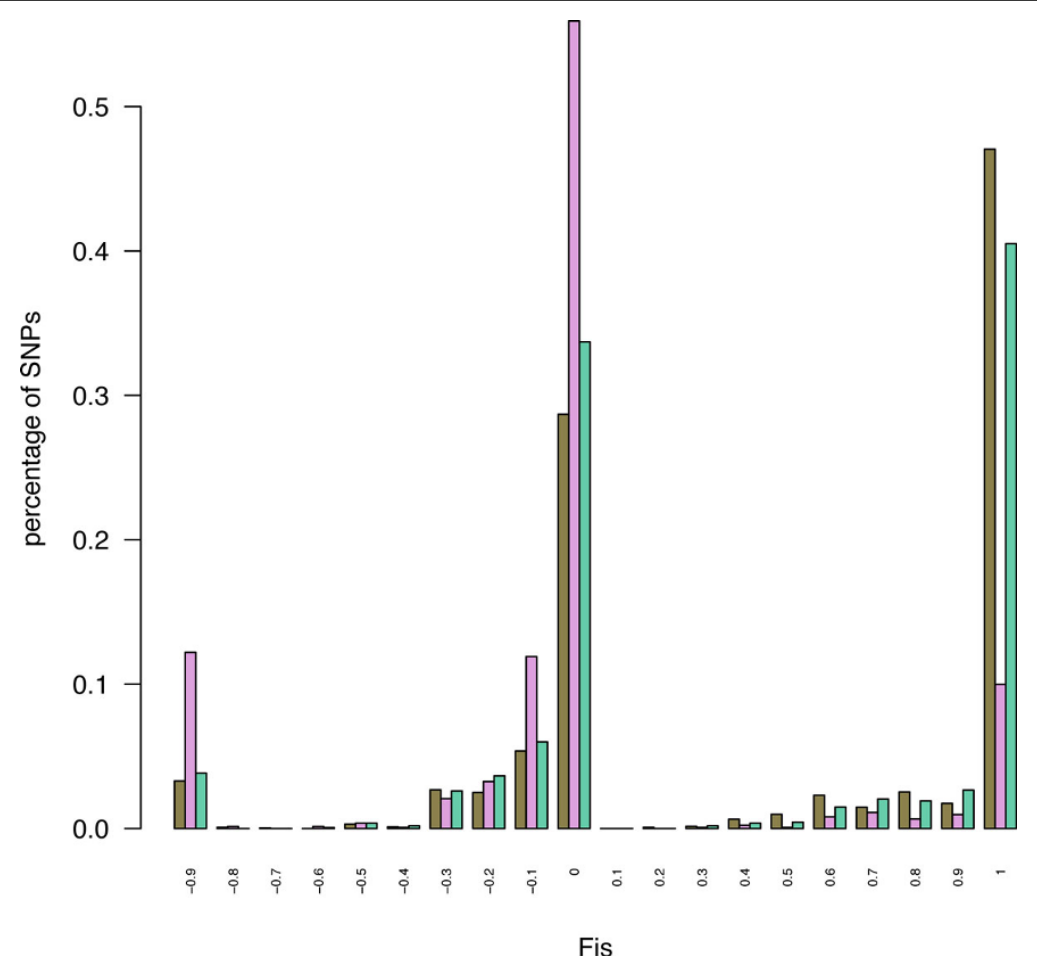

Figure 3 Distribution of Fis values of: diploid SNPs (brown), de novo SNPs (pink) and HomeoSplitter SNPs (green). Fis values were calculated as the heterozygosity deficit relative to panmixia. Allelic frequencies were estimated from the called genotypes with high confidence values (see text). Negative Fis values suggest fixed divergence between homeologous/paralogous copies. Fis around 0 indicate possible mixture of homeology and intra genome polymorphism. Fis values close to 1 sign a priori intra genome polymorphic sites. See Figure 1 for the pipeline leading to the detection of these three SNP sets

188 homologous SNPs out of the 1,360 SNPs detected in the mapping on the de novo durum contigs, i.e., only a relative fraction of $13.8 \%$. This ratio rocketed to $47 \%$ $(762 / 1620)$ in the mapping on the HomeoSplitter

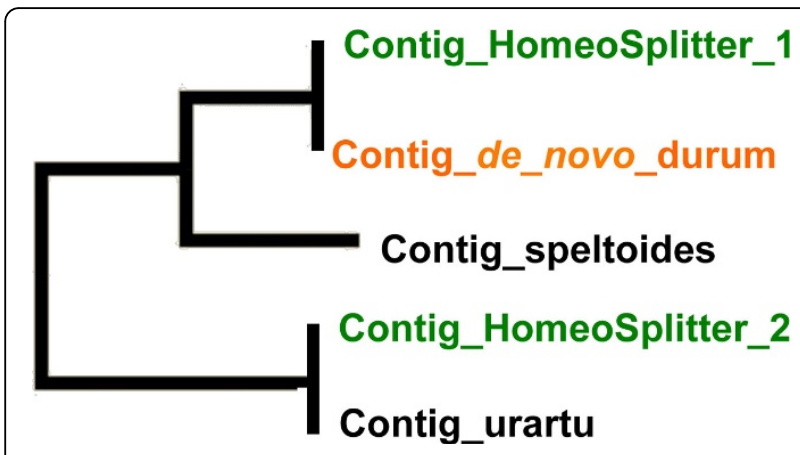

Figure 4 Phylogenetic tree inferred on homologous contigs from cluster 6960 . The original contig obtained by de novo assembling (contig_de_novo_durum) was more similar to the speltoides contig (contig_speltoides) than to the urartu one (contig_urartu). After using HomeoSplitter we obtained the most expressed split contig (contig_HomoeoSplitter_1) that is still similar to the speltoides one and one less expressed contig

(contig_HomoeoSplitter_2) highly similar to the urartu one. contigs, reasonably close to the ratio obtained on mapping on the diploid contigs, i.e., 56\% (1507/2661). A slight superiority of HomeoSplitter compared to MajoritySplitter is observed, the percentage of valuable homologous detection is only $45 \%$ in the latter case $(710 /$ 1575) but this difference is not significant (chi2 $=1.23$, $d f=1, p$-value $=0.73)$.

The discrepancy between the absolute numbers of SNPs detected in mapping on diploid $(2,601)$ and on HomeoSplitter contigs $(1,360)$ may be due to a difference of the considered fraction of the transcriptome. Indeed, on the 2,505 clusters of the benchmark, the summed length of contigs is $\sim 4.85 \mathrm{Mb}$ in diploids and only $\sim 1.89 \mathrm{Mb}$ in HomeoSplitter contigs (38.9\%).

\section{Conclusion}

HomeoSplitter relies on a straightforward algorithm based on nucleotide counts. This leads to an extremely efficient solution to deal with thousands of contigs in a matter of minutes. HomeoSplitter is efficient to rebuild two likely homeologous sequences from contigs on which mapping generates a large excess of heterozygous genotypes. Remapping reads on these new sequences leads to a huge gain of SNPs with a priori correct genotyping properties 
(Fis >0.6). In our benchmark, HomeoSplitter detects valuable SNPs for $47 \%$ of the detected polymorphism compared to the $13.8 \%$ initial mapping, the latter case being close to the ratio previously reported in the allo-tetraploid rapeseed [3]. This efficiency is only slightly inferior to the mapping on the diploid references (56\%).

HomeoSplitter is expected to be efficient when a bias in the expression between homeologous copies exists in at least some accessions, whatever its distribution among accessions. Its power is strongly reduced in case of balanced expression. One planned extension is to warn the user in such cases that can be identified since several splits have almost identical likelihood. Furthermore, HomeoSplitter offers the possibility to seek for specific genome polymorphism to develop flanking primers for SNPs to be developed as markers on high throughput micro-arrays, hence possibly improving the genotype calling [8].

Computation time of HomeoSplitter is short and is thus a good complement of the approach relying on diploid reference transcriptomes [1,9]. Indeed, some genes may not be expressed or detected in one or the two diploid transcriptomes (or absent in the diploid genomes due to gene deletions subsequent to polyploidisation). In our case, since the reads number used for assembling transcriptomes was rather low, the benchmark including clusters of speltoides, urartu and durum contigs concerns only $\sim 10 \%$ of the durum wheat contigs. HomeoSplitter appears as a convenient method for treating the remaining $90 \%$. HomeoSplitter also avoids the fine tuning of stringency used for de novo assembly [1] and allows the use of a relatively permissive similarity for the assembly. This permits to keep all homologous alleles within the same contig, and to be able to examine the excess of heterozygosity a posteriori. As the divergence may vary a lot among genes (here the average similarity between urartu and speltoides is $97.5 \%$ with a standard deviation of $1.03 \%$ ), a fine tuning of stringency is difficult to determine for a whole transcriptome (or genome).

Questionable sites were easily detected in the selfing durum wheat. For outbred species, the questionable sites may be detected by the likelihood approach used in the read2snp package [5]. It compares the likelihood of data for the existence of paralogs in diploid species with any kind of mating system. It may be further included in future version of HomeoSplitter. HomeoSplitter does not exploit the individual paired-end read information as in phasing methods [18-20]. A pipeline to disentangle homeologous copies based on such diploid phasing methods has just been published [37]. This method does not especially target questionable sites and does not exploit the well-documented phenomenon of expression level dominance in homeologous copies [26-28]. Mixing both approaches seems a promising solution; but HomeoSplitter offers already a rapid, simple and efficient solution.

\section{Additional material}

Additional file 1: This text file provides further details about the plant material.

Additional file 2: This text file provides further details about RNA extraction and sequencing protocols.

Additional file 3: This text file provides further details about contig assembly, read mapping and SNPs identifications.

\section{Competing interests}

The authors declare that they have no competing interests.

\section{Authors' contributions}

MTP and JD designed and managed the plant experiments. MA and SS prepared cDNA libraries from plant samples. GS and YH processed NGS data from raw reads to mapping. JD, VR, YH and SG conceived the homeoSplitter method, VR implemented it and $Y H$ conducted validation analysis. VR, MTP, JD, SG and SS wrote the paper, all authors read and approved the final version.

\section{Acknowledgements}

This work was partially funded by the French Agence Nationale de la Recherche Investissements d'avenir / Bioinformatique: ANR-10-BINF-01-02, Ancestrome. YH was granted by the flagship project Agropolis Resource Center for Crop Conservation, Adaptation and Diversity (ARCAD) funded by the Agropolis Fondation. INRA funded the data production through its Actions Incitatives program (EPO project). Olivier Guillaume and Aziz Moussa also contributed, through short student projects, to this work. Sequencing was kindly performed on the HiSeq2000 sequencer at the Montpellier Genomix (http://www.mgx.cnrs.fr) sequencing facility. We are grateful to the three anonymous referees whose comments helped us to improve this paper. This publication is contribution 2013-118 of the Institut des Sciences de l'Evolution de Montpellier (UMR 5554CNRS).

\section{Declarations}

The publication costs for this article were funded by the Agropolis Fondation under the ARCAD projet No 0900-001.

This article has been published as part of BMC Bioinformatics Volume 14 Supplement 15, 2013: Proceedings from the Eleventh Annual Research in Computational Molecular Biology (RECOMB) Satellite Workshop on Comparative Genomics. The full contents of the supplement are available online at http://www.biomedcentral.com/bmcbioinformatics/supplements/ $14 / \$ 15$.

\section{Authors' details}

${ }^{1}$ Montpellier SupAgro, UMR AGAP, F-34060 Montpellier, France. ${ }^{2}$ INRA, UMR AGAP, F-34060 Montpellier, France. ${ }^{3}$ Institut des Sciences de l'Evolution de Montpellier (ISE-M), UMR 5554 CNRS Université Montpellier II, place E. Bataillon, CC 064, 34095 Montpellier cedex 05, France.

Published: 15 October 2013

\section{References}

1. Kaur S, Francki MG, Forster JW: Identification, characterization and interpretation of single-nucleotide sequence variation in allopolyploid crop species. Plant biotechnology journal 2012, 10:125-138.

2. Barbazuk WB, Emrich SJ, Chen HD, Li L, Schnable PS: SNP discovery via 454 transcriptome sequencing. The Plant journal 2007, 51:910-918.

3. Trick M, Long Y, Meng J, Bancroft I: Single nucleotide polymorphism (SNP) discovery in the polyploid Brassica napus using Solexa transcriptome sequencing. Plant biotechnology journal 2009, 7:334-346.

4. Ozsolak F, Milos PM: RNA sequencing: advances, challenges and opportunities. Nat Rev Genet 2011, 12(2):87-98. 
5. Gayral P, Melo-Ferreira J, Glémin S, Bierne N, Carneiro M, Nabholz B, Lourenco JM, Alves PC, Ballenghien M, Faivre N, et al: Reference-free population genomics from next-generation transcriptome data and the vertebrate-invertebrate gap. PLOS genetics 2013, 9:e1003457.

6. Leitch IJ, Bennett MD: Polyploidy in angiosperms. Trends in Plant Science 1997, 2:470-476

7. Salse J: In silico archeogenomics unveils modern plant genome organisation, regulation and evolution. Curr Opin Plant Biol 2012, 15(2):122-130.

8. Trebbi D, Maccaferri M, de Heer P, Sørensen A, Giuliani S, Salvi S, Sanguineti MC, Massi A, van der Vossen EAG, Tuberosa R: High-throughput SNP discovery and genotyping in durum wheat (Triticum durum Desf.). Theoretical and applied genetics 2011, 123:555-569.

9. Udall JA, Swanson JM, Nettleton D, Percifield RJ, Wendel JF: A nove approach for characterizing expression levels of genes duplicated by polyploidy. Genetics 2006, 173(3):1823-1827.

10. Clark AG: Inference of haplotypes from PCR-amplified samples of diploid populations. Mol Biol Evol 1990, 7(2):111-122.

11. Browning SR, Browning BL: Haplotype phasing: existing methods and new developments. Nat Rev Genet 2011, 12(10):703-714

12. Graca A, Lynce I, Marques-Silva J, Oliveira AL: Haplotype inference by Pure Parsimony: a survey. J Comput Biol 2010, 17(8):969-992.

13. Irurozki E, Calvo B, Lozano JA: A preprocessing procedure for haplotype inference by pure parsimony. IEEE/ACM Trans Comput Biol Bioinform 2011 8(5):1183-1195.

14. Huang $Y T$, Chao KM, Chen T: An approximation algorithm for haplotype inference by maximum parsimony. J Comput Biol 2005, 12(10):1261-1274.

15. Excoffier $L$, Slatkin M: Maximum-likelihood estimation of molecular haplotype frequencies in a diploid population. Mol Biol Evol 1995, 12(5):921-927.

16. Niu T, Qin ZS, Xu X, Liu JS: Bayesian haplotype inference for multiple linked single-nucleotide polymorphisms. Am J Hum Genet 2002, 70(1):157-169.

17. Stephens M, Donnelly P: A comparison of bayesian methods for haplotype reconstruction from population genotype data. Am J Hum Genet 2003, 73(5):1162-1169.

18. Long Q, MacArthur D, Ning Z, Tyler-Smith C: HI: haplotype improver using paired-end short reads. Bioinformatics 2009, 25(18):2436-2437.

19. Aguiar D, Istrail S: HapCompass: a fast cycle basis algorithm for accurate haplotype assembly of sequence data. J Comput Biol 2012, 19(6):577-590.

20. Bansal V, Bafna V: HapCUT: an efficient and accurate algorithm for the haplotype assembly problem. Bioinformatics 2008, 24(16):1153-159.

21. Sasaki E, Sugino RP, Innan H: The linkage method: a novel approach for SNP detection and haplotype reconstruction from a single diploid individual using next generation sequence data. Mol Biol Evol 2013.

22. Iqbal Z, Caccamo M, Turner I, Flicek P, McVean G: De novo assembly and genotyping of variants using colored de Bruijn graphs. Nat Genet 2012, 44(2):226-232.

23. de Bruijn NG: A Combinatorial Problem. Koninklijke Nederlandse Akademie v Wetenschappen 1946, 49:758-764.

24. Iqbal Z, Turner I, McVean G: High-throughput microbial population genomics using the Cortex variation assembler. Bioinformatics 2013, 29(2):275-276

25. Giorgi D, Farina A, Grosso V, Gennaro A, Ceoloni C, Lucretti S: FISHIS: fluorescence in situ hybridization in suspension and chromosome flow sorting made easy. PLoS One 2013, 8(2):e57994.

26. Adams KL: Evolution of duplicate gene expression in polyploid and hybrid plants. J Hered 2007, 98(2):136-141

27. Yoo MJ, Szadkowski E, Wendel JF: Homoeolog expression bias and expression level dominance in allopolyploid cotton. Heredity (Edinb) 2013, 110(2):171-180.

28. Rapp RA, Udall JA, Wendel JF: Genomic expression dominance in allopolyploids. BMC Biol 2009, 7:18

29. Dvořák J, di Terlizzi $P$, Zhang H-B, Resta P: The evolution of polyploid wheats: identification of the A genome donor species. Genome 1993, 36(1):21-31.

30. Dvořák J, Zhang HB: Variation in repeated nucleotide sequences sheds light on the phylogeny of the wheat B and G genomes. Proc Natl Acad Sci USA 1990, 87:9640-9644

31. Huang S, Sirikhachornkit A, Su X, Faris J, Gill B, Haselkorn R, Gornicki P: Genes encoding plastid acetyl-CoA carboxylase and 3-phosphoglycerate kinase of the Triticum/Aegilops complex and the evolutionary history of polyploid wheat. Proc Natl Acad Sci USA 2002, 99(12):8133-8138.

32. Dvořák J, Akhunov ED: Tempos of gene locus deletions and duplications and their relationship to recombination rate during diploid and polyploid evolution in the Aegilops-Triticum alliance. Genetics 2005, 171:323-332

33. Wright S: Genetical structure of populations. Nature 1950, 166(4215): 247-258.

34. Huang X: CAP3: A DNA Sequence Assembly Program. Genome Research 1999, 9:868-877.

35. Gayral P, Melo-Ferreira J, Glemin S, Bierne N, Carneiro M, Nabholz B, Lourenco JM, Alves PC, Ballenghien M, Faivre N, et al: Reference-free population genomics from next-generation transcriptome data and the vertebrate-invertebrate gap. PLoS Genet 2013, 9(4):e1003457.

36. Li H, Handsaker B, Wysoker A, Fennell T, Ruan J, Homer N, Marth G, Abecasis G, Durbin R: The Sequence Alignment/Map format and SAMtools. Bioinformatics 2009, 25(16):2078-2079.

37. Krasileva KV, Buffalo V, Bailey P, Pearce S, Ayling S, Tabbita F, Soria M, Wang S, Consortium I, Akhunov $E$, et al: Separating homeologs by phasing in the tetraploid wheat transcriptome. Genome Biol 2013, 14(6):R66.

38. Thuillet AC, Bataillon T, Poirier S, Santoni S, David JL: Estimation of longterm effective population sizes through the history of durum wheat using microsatellite data. Genetics 2005, 169:1589-1599.

39. Haudry A, Cenci A, Ravel C, Bataillon T, Brunel D, Poncet C, Hochu I, Poirier S, Santoni S, Glémin S, et al: Grinding up wheat: a massive loss of nucleotide diversity since domestication. Mol Biol Evol 2007, 24:1506-1517.

40. Cavanagh CR, Chao S, Wang S, Huang BE, Stephen S, Kiani S, Forrest K, Saintenac C, Brown-Guedira GL, Akhunova A, et al: Genome-wide comparative diversity uncovers multiple targets of selection for improvement in hexaploid wheat landraces and cultivars. Proc Natl Acad Sci USA 2013, 110:8057-8062

41. Martin M: Cutadapt removes adapter sequences from high-throughput sequencing reads. EMBnet 2011, 17:10-12

42. Simpson JT, Wong K, Jackman SD, Schein JE, Jones SJM, Birol I: ABySS: a parallel assembler for short read sequence data. Genome research 2009, 19:1117-1123.

43. Li H, Durbin R: Fast and accurate short read alignment with BurrowsWheeler transform. Bioinformatics 2009, 25:1754-1760.

doi:10.1186/1471-2105-14-S15-S15

Cite this article as: Ranwez et al: Disentangling homeologous contigs in allo-tetraploid assembly: application to durum wheat. BMC Bioinformatics 2013 14(Suppl 15):S15

\section{Submit your next manuscript to BioMed Central and take full advantage of:}

- Convenient online submission

- Thorough peer review

- No space constraints or color figure charges

- Immediate publication on acceptance

- Inclusion in PubMed, CAS, Scopus and Google Scholar

- Research which is freely available for redistribution
Biomed Central 